\title{
Supporting Information \\ Dynamic tuning of gap plasmon resonances using a solid-state electrochromic device
}

\author{
Yiyang $\mathrm{Li}^{1,2 \uparrow^{*}}$, Jorik van de Groep ${ }^{3 \dagger}$, A. Alec Talin ${ }^{1}$, Mark L. Brongersma ${ }^{3 *}$ \\ ${ }^{1}$ Sandia National Laboratories, Livermore, CA 94550 \\ ${ }^{2}$ Department of Materials Science and Engineering, Stanford University, Stanford, CA 94305 \\ ${ }^{3}$ Geballe Laboratory of Advanced Materials, Stanford University, Stanford, CA 94305 \\ ${ }^{\dagger}$ Equal contribution \\ *brongersma@stanford.edu; *yiyli@sandia.gov
}

\section{Sample fabrication for ellipsometric measurements}

The ellipsometry measurements are conducted on $\mathrm{WO}_{3}$ films sputtered on $\mathrm{Si}$, then lithiated in a liquid electrochemical cell. 100-mm-diameter, $500 \mu \mathrm{m}$-thick doped Si wafers (resistance 1-5 $\mathrm{Ohm}-\mathrm{cm}$ ) are used as the substrate without further processing. $\mathrm{WO}_{3}$ is deposited on the Si substrate through reactive sputtering with an 8-target Kurt J. Lesker LAB Line Sputter 8 thin film deposition system. Here, the target was a 2 -inch W target, the DC power was $200 \mathrm{Watt}$, and the sputter environment was a 5 mtorr background pressure with $50 \%$ Ar and $50 \% \mathrm{O}_{2}$, controlled by mass flow controllers. Our films are sputtered in $4 \mathrm{~min}$ and yielded 64-nm-thick films, measured through ellipsometry and X-ray reflection analysis. After sputtering, the substrate is cut into 1-cm squares using a DISCO wafer saw. The "pristine" sample is not processed any further.

The "lithiated" and "delithiated" samples are further prepared electrochemically. The 1-cm squares are placed into CR2016 "coin cells"; electrochemical cells that contained two stainless steel plates around a polypropylene gasket. Each cell contains the $\mathrm{WO}_{3}$ film deposited on $\mathrm{Si}$, two Celgard separators, and a Li metal anode, and is flooded with Gotion LP40 electrolyte containing $1 \mathrm{M} \mathrm{LiPF}_{6}$ salt dissolved in an ethylene carbonate/diethyl carbonate solvent. The devices are cycled using cyclic voltammetry (Fig. S2) for $\sim 3$ cycles between $2 \mathrm{~V}$ and $3.5 \mathrm{~V}$ vs the counter-electrode, Li. Afterwards, the lithiated sample is held at $2 \mathrm{~V}$ for $1 \mathrm{hr}$, and the delithiated sample is held at 2 $\mathrm{V}$ for $30 \mathrm{~min}$ and at $4 \mathrm{~V}$ for $1 \mathrm{hr}$. Both cells are disassembled in an Ar glovebox, where the $\mathrm{WO}_{3}$ films on the Si substrates are harvested. The films are rinsed with anhydrous dimethyl carbonate, dried under Ar, and placed aluminum-coated heat-seal pouches. The sample is not exposed to ambient atmosphere between the disassembly and the ellipsometry, except for $\sim 1$ min during the ellipsometry measurement.

Ellipsometry are performed using a Woollam M2000 system at incident angles between $45^{\circ}$ and $75^{\circ}$, with $3^{\circ}$ intervals, using incident light between $\lambda=280 \mathrm{~nm}$ and $\lambda=1500 \mathrm{~nm}$. The pristine data is fitted using a Tauc-Lorentz model, and generally consistent with literature, while the lithiated and delithiated results were fitted using a combination of a Tauc-Lorentz and a Drude term. 


\section{Fabrication of $\mathrm{Al} / \mathrm{WO} \mathrm{O}_{3} / \mathrm{Al}$ gap plasmon structures and the electrochemical cell}

The gap plasmon structures, schematically shown in Fig. 1b, are fabricated on Si wafers with a 500-nm wet thermal oxide layer. Optical photolithography using S1813 photoresist and a Karl Suss contact aligner are used to define the Al bottom layer and the $\mathrm{WO}_{3}$ layer. 100-nm-thick $\mathrm{Al}$ is grown using thermal evaporation, and $17-\mathrm{nm}$-thick $\mathrm{WO}_{3}$ is grown using reactive sputtering (see previous section). Excess material is removed using lift-off in acetone. Electron-beam lithography using a bi-layer PMMA resist and a JEOL 6300-FS $100 \mathrm{kV}$ system is used to define the top layer of $\mathrm{Al}$ gap plasmon resonators above the $\mathrm{WO}_{3} .50 \mathrm{~nm}$ of $\mathrm{Al}$ is deposited using electronbeam evaporation. Excess material is removed using lift-off in acetone. This completes the fabrication of the gap plasmon structures, where the colors depend on the dimensions of the structures and the incident light polarization.

To complete the electrochemical cell, excess $\mathrm{Li}_{0.7} \mathrm{FePO}_{4}$ particles are drop-cast on an $\mathrm{Al}$ current collector about $5 \mathrm{~mm}$ away from the $\mathrm{WO}_{3}$ patterns. The $\mathrm{Li}_{0.7} \mathrm{FePO}_{4}$ and $\mathrm{WO}_{3}$ are electrically isolated from each other via the thermal oxide. These $\mathrm{Li}_{0.7} \mathrm{FePO}_{4}$ particles are chemically delithiated from pristine $\mathrm{LiFePO}_{4}$ using a stoichiometric quantity of potassium persulfate $\left(\mathrm{K}_{2} \mathrm{~S}_{2} \mathrm{O}_{8}\right)$ dissolved in water. As a two-phase material, $\mathrm{Li}_{0.7} \mathrm{FePO}_{4}$ has a flat voltage plateau to serve as a reliable reference electrode; the amount of lithium in $\mathrm{Li}_{0.7} \mathrm{FePO}_{4}$ is significantly larger than the amount that can be inserted into $\mathrm{WO}_{3}$. The polymer electrolyte is then spun-coat on the sample with a thickness of $\sim 2 \mu \mathrm{m}$, and ionically connects the $\mathrm{WO}_{3}$ to the $\mathrm{Li}_{0.7} \mathrm{FePO}_{4}$ electrodes. The shape of the cyclic voltammetry curve (Fig. 3a) is qualitatively similar with the one cycled in the liquid electrolytes, only with a different reference electrode.

\section{Filtering of Fabry-Perot resonances}

The Fabry-Perot resonances that arise from the finite $\sim 2-\mu \mathrm{m}$-thick electrolyte films are digitally filtered in Matlab using a six-order Butterworth filter. The comparison of the filtered and unfiltered results is shown in Fig. S5.

\section{Finite-difference time-domain simulations}

FDTD simulations are performed using commercial software (Lumerical FDTD) ${ }^{1}$. Aluminum (Al) particles with rounded corners (10 nm radius), $50 \mathrm{~nm}$ height, $60 \mathrm{~nm}$ width, and variable length are placed on top of a $17 \mathrm{~nm} \mathrm{WO}_{3}$ layer and a semi-infinite $\mathrm{Al}$ substrate. The background refractive index is set to 1.45 to simulate the solid electrolyte. A plane wave $(\lambda=400-800 \mathrm{~nm})$ is used as a source. A 2D flux monitor is placed above the source and used to measure the reflection. A simulation box with $225 \mathrm{~nm}$ dimensions in the $\mathrm{x}-\mathrm{y}$ plane and periodic boundary conditions is used to simulate a periodic array. A perfectly-matching layers (PML) boundary condition is used on the top, and a metal boundary condition positioned inside the Al substrate at the bottom to simulate a semi-infinite substrate. Around the particle, a $1 \mathrm{~nm}$ refinement mesh is used. Optical constants for the $\mathrm{WO}_{3}$ are obtained from spectroscopic ellipsometry (Fig. S6), and the optical constants for Al are taken from Palik $^{2}$.

\section{Optical measurements}

The optical reflection spectra are measured using a Nikon C2 confocal microscope. The samples are illuminated (wide field with aperture and focal stop closed) using a white-light halogen lamp. A 50x long-working distance objective (Nikon LU Plan, $0.55 \mathrm{NA}, 10.1 \mathrm{~mm}$ working distance) is used in combination with a $90 \mu \mathrm{m}$ pinhole to locally collect the reflected light, which is then analyzed using a SpectraPro 2300i spectrometer (150 lines $/ \mathrm{mm}$, blazed for $\lambda=500 \mathrm{~nm}$ ) and PIXIS 
silicon CCD camera $\left(-70{ }^{\circ} \mathrm{C}\right.$ detector temperature). The polarization is controlled using a broad band wire grid polarizer in the detection path. The reported spectra are the average of 25 frames $(0.8 \mathrm{~s}$ integration time each) and normalized to the spectrum of a protected silver mirror (Thorlabs PF10-03-P01) to correct for the system response.

\section{Supplemental figures}

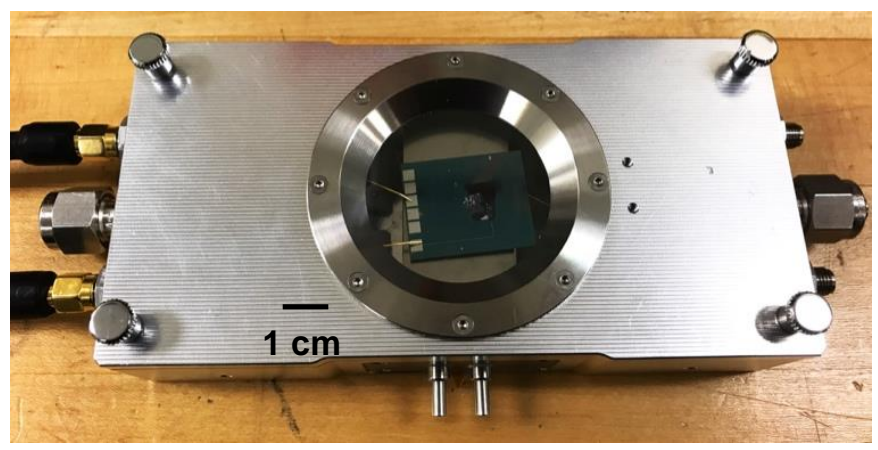

Figure S1: Environmental housing used for optical characterization. The environmental chamber (NEXTRON) enables the sample to be housed in an insert Argon environment at a controlled temperature. The electrical controls enable optical switching.

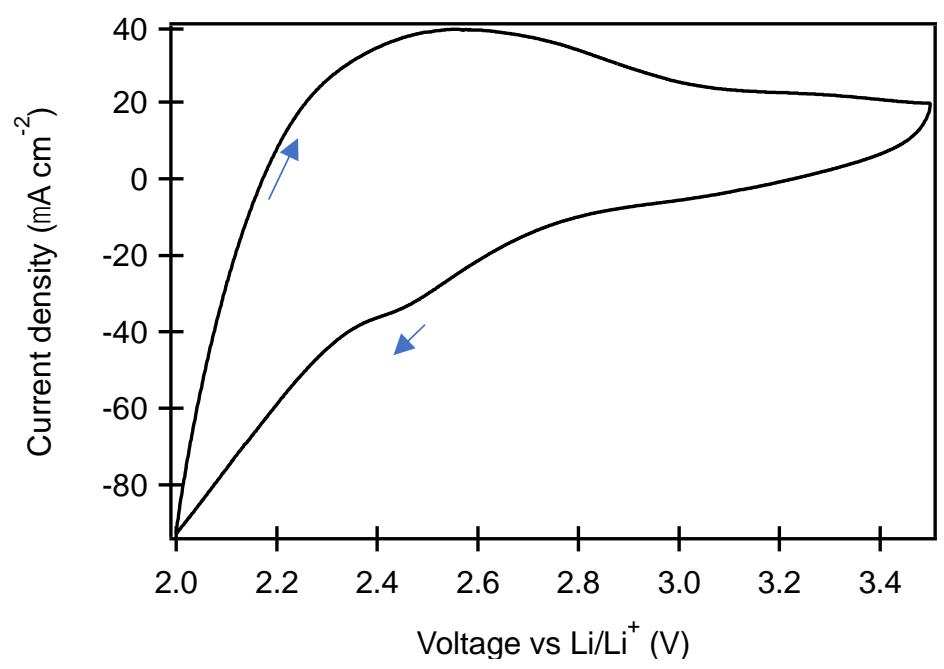

Figure S2: Cyclic voltammetry of a 166-nm-thick $\mathrm{LixWO}_{3}$ chip grown on Si. The counterelectrode is a Li metal foil. The scan rate is $3 \mathrm{mV} \mathrm{s}^{-1}$. We use coulomb counting to obtain the lithium concentration upon lithiation by taking the time integral of the current density to obtain the total amount of charge transferred (in coulombs), computing the number of moles of lithium, and then normalizing that by the number of $\mathrm{WO}_{3}$ sites to obtain the lithium concentration $X$ assuming a density of $7 \mathrm{~g} \mathrm{~cm}^{-3}$. Because this measurement was conducted for materials characterization, we referenced it against $\mathrm{Li} / \mathrm{Li}^{+}$, whereas the voltage is referenced against $\mathrm{Li}_{0.7} \mathrm{FePO}_{4} / \mathrm{Li}^{+}$in all other figures. To convert from a $\mathrm{Li} / \mathrm{Li}^{+}$to a $\mathrm{Li}_{0.7} \mathrm{FePO}_{4} / \mathrm{Li}^{+}$reference, it is 
necessary to subtract $3.4 \mathrm{~V}$ from the voltage here because $\mathrm{Li}_{0.7} \mathrm{FePO}_{4}$ has an electrochemical potential of $3.4 \mathrm{~V}$ against $\mathrm{Li} / \mathrm{Li}^{+}$. For example, $2.0 \mathrm{~V}$ here is equivalent to $-1.4 \mathrm{~V}$ in Fig. $3 \mathrm{a}$.

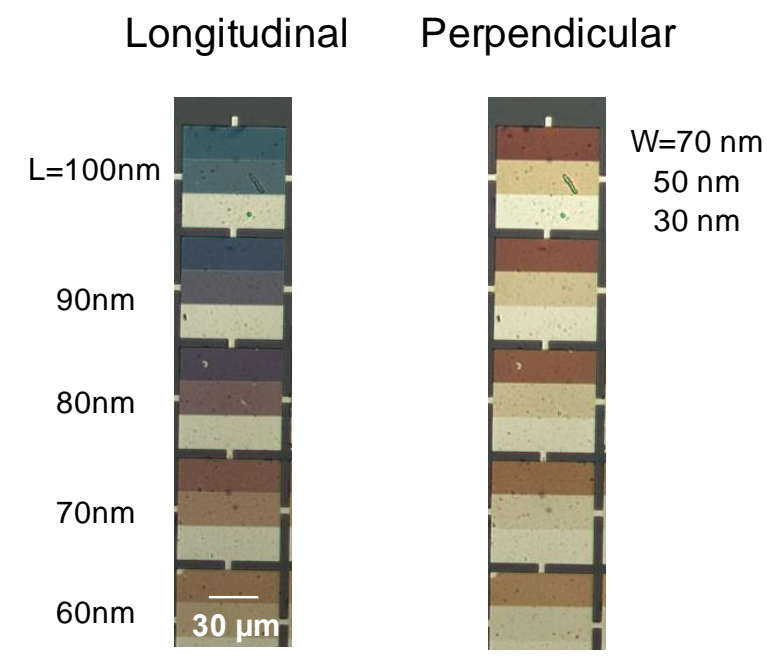

Figure S3: Optical micrograph of $\mathrm{Al} / \mathrm{WO}_{3} / \mathrm{Al}$ gap plasmon structure containing different sized Al particles with a length $\boldsymbol{L}$ and a width $\boldsymbol{W}$. In the longitudinal polarization, the structural color is highly dependent on the length $L$. In the perpendicular polarization, the structural color has almost no dependence on the length $L$, and is instead related to the width $W$. 


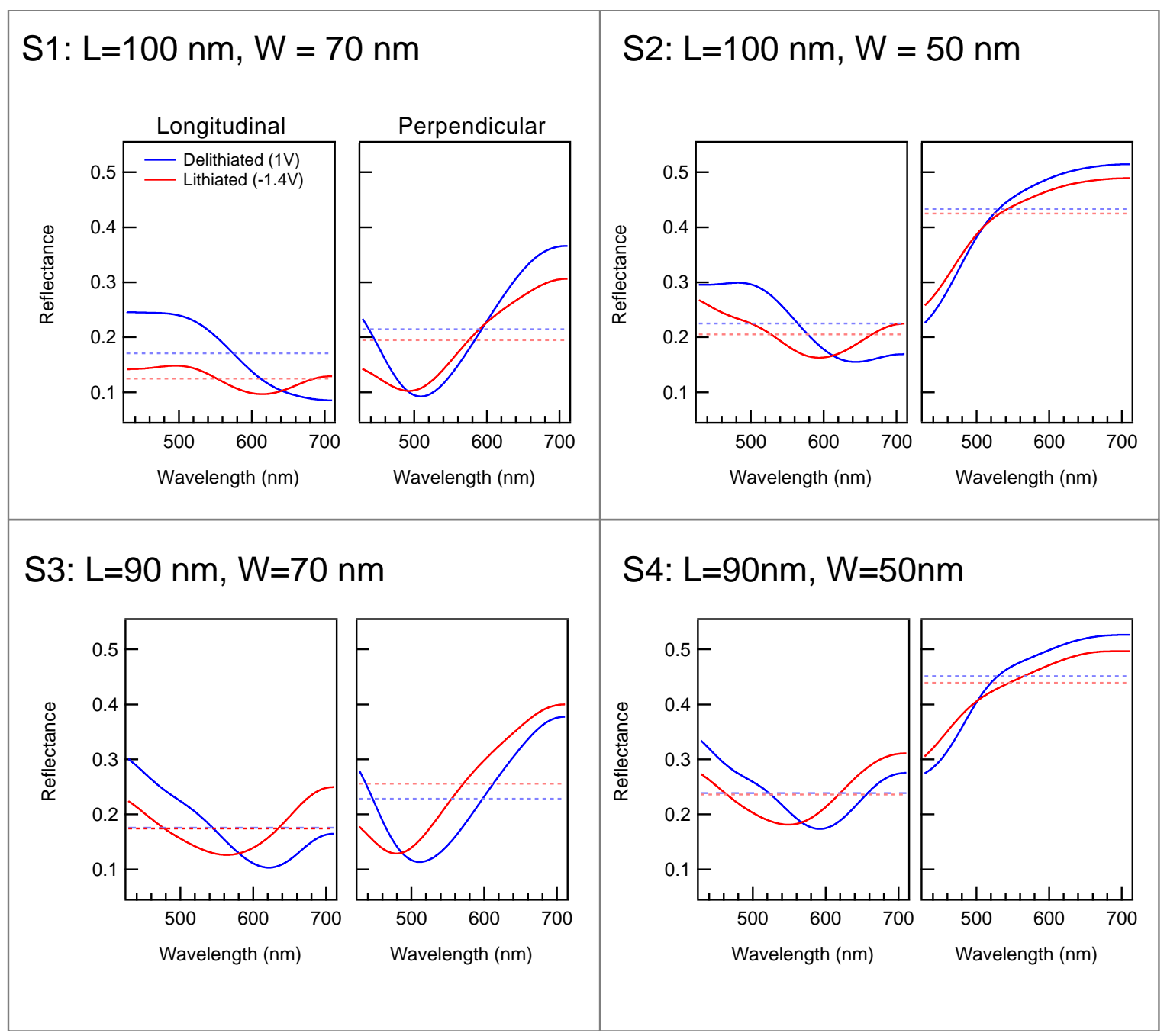

Figure S4: Reflectance spectra for four representative patterns upon lithiation and delithiation. The length $L$ and width $W$ of the Al nanoparticles varies between the patterns. All spectra show a blueshift for the reflectance minimum spectra upon lithiation. The red and blue dashed lines indicate the magnitude of the reflectance averaged over all wavelengths. The attenuation magnitude of 0.01 is calculated by taking the average of $\bar{R}_{D}-\bar{R}_{L}$ across all samples, where $\bar{R}_{L}$ and $\bar{R}_{D}$ are the measured reflectance of the lithiated and delithiated spectra averaged across all wavelengths. The attenuation percentage of $5 \%$ is calculated through $1-\frac{\bar{R}_{L}}{\bar{R}_{D}}$, and indicates minimal attenuation. 

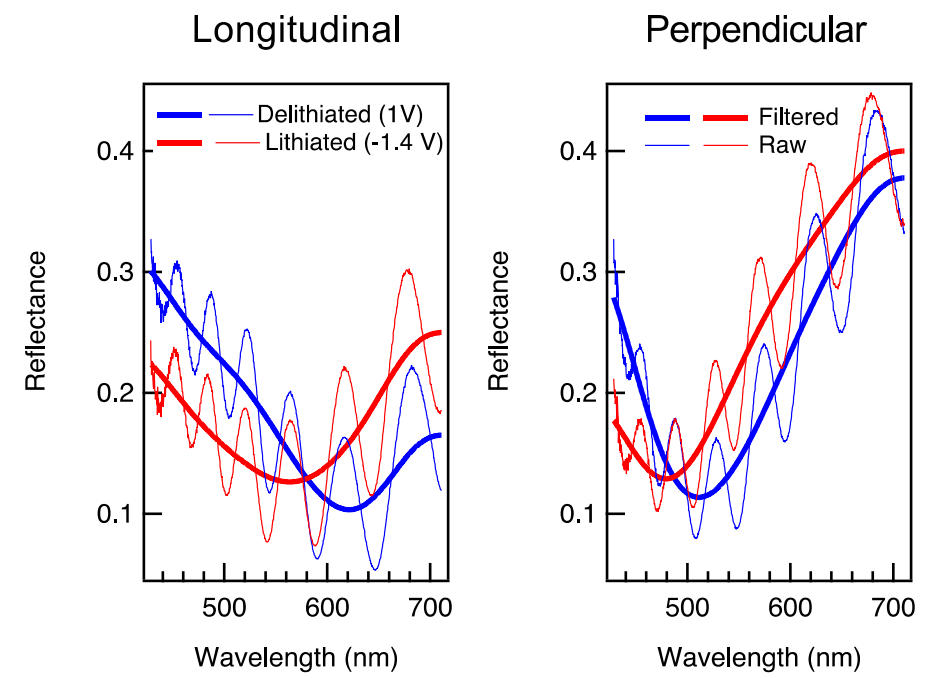

Figure S5: Filtering the Fabry-Perot fringes. The Fabry-Perot fringes arise from the $\sim 2 \mu \mathrm{m}-$ thick solid polymer electrolyte. A sixth-order low-pass Butterworth filter is used to remove the fringes to better visualize the underlying reflection features. 

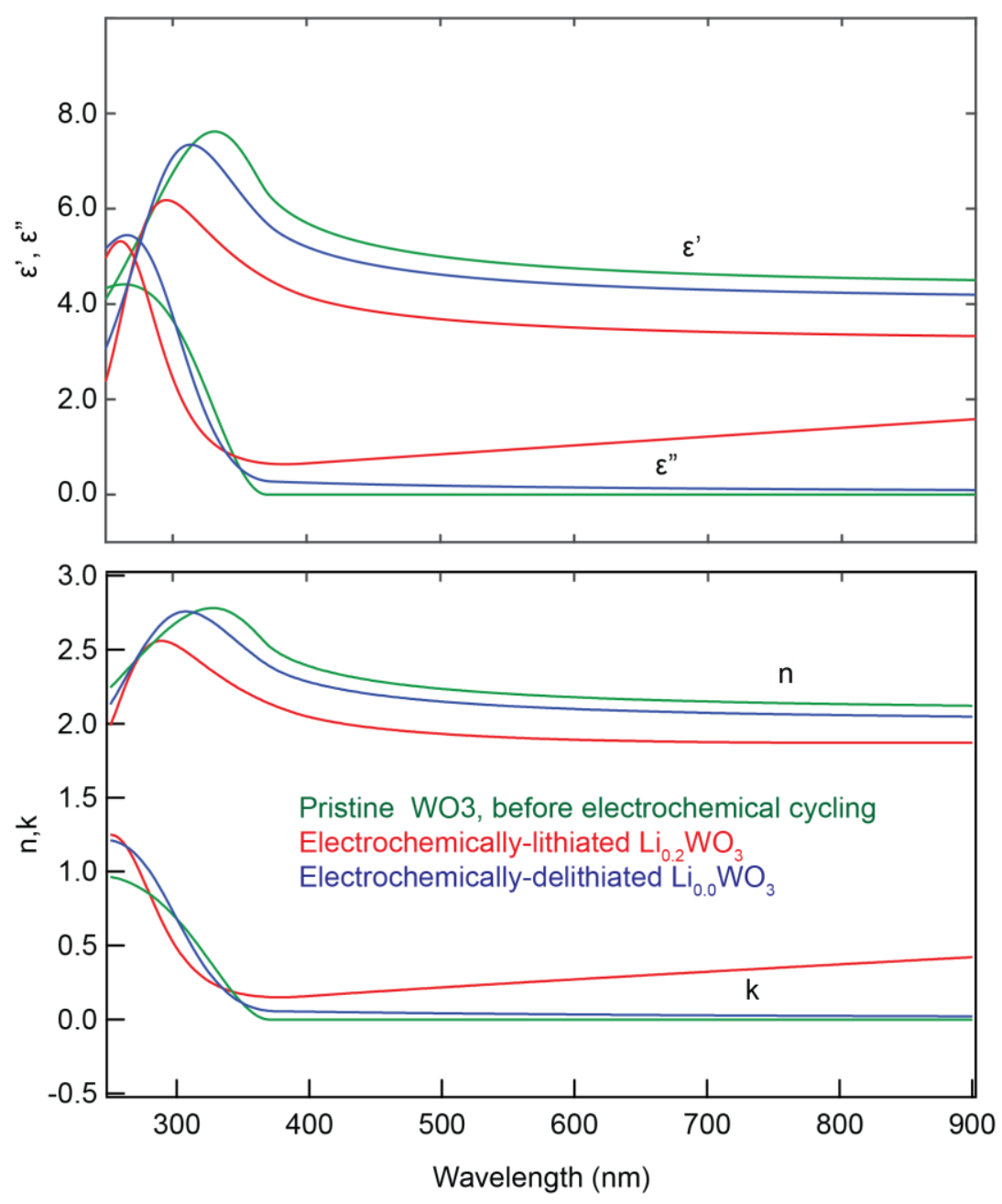

Figure S6: Optical constants of pristine, electrochemically lithiated, and electrochemically delithiated sample. Relative permittivity (top) and refractive index (bottom). The pristine $\mathrm{WO}_{3}$ is not processed after the sputter deposition, while the lithiated and delithiated samples are electrochemically cycled and then held at a voltage of $2.0 \mathrm{~V}$ vs $\mathrm{Li}$ and $4.0 \mathrm{~V}$ vs Li for $\sim 30 \mathrm{~min}$ before measurement. The electrochemically delithiated $\mathrm{Li}_{0.0} \mathrm{WO}_{3}$ almost fully recovers the refractive index of the pristine, as deposited $\mathrm{WO}_{3}$. Deviations are attributed to some residual lithium in the structure. FDTD simulations are conducted using the optical constants for the electrochemically-lithiated and electrochemically-delithiated films, not the pristine material. 
a

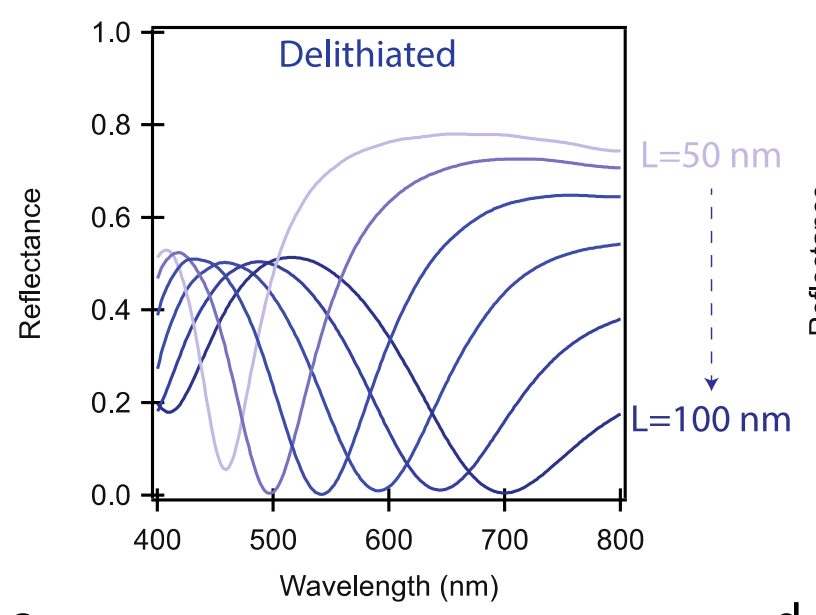

C

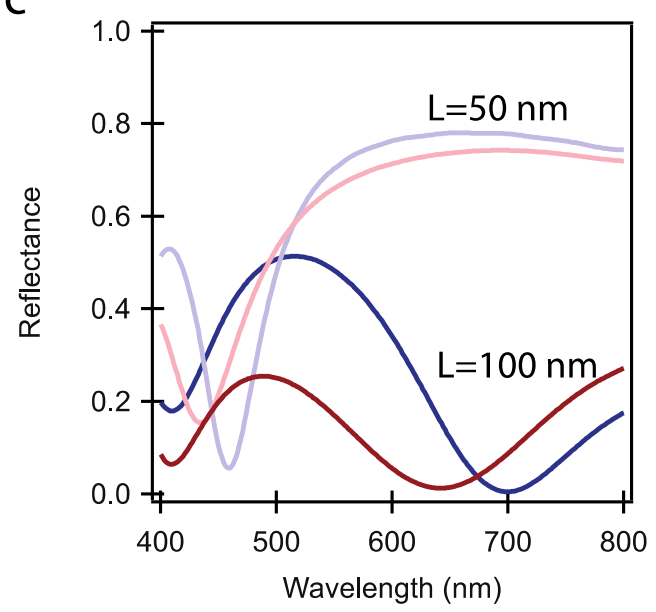

b

d
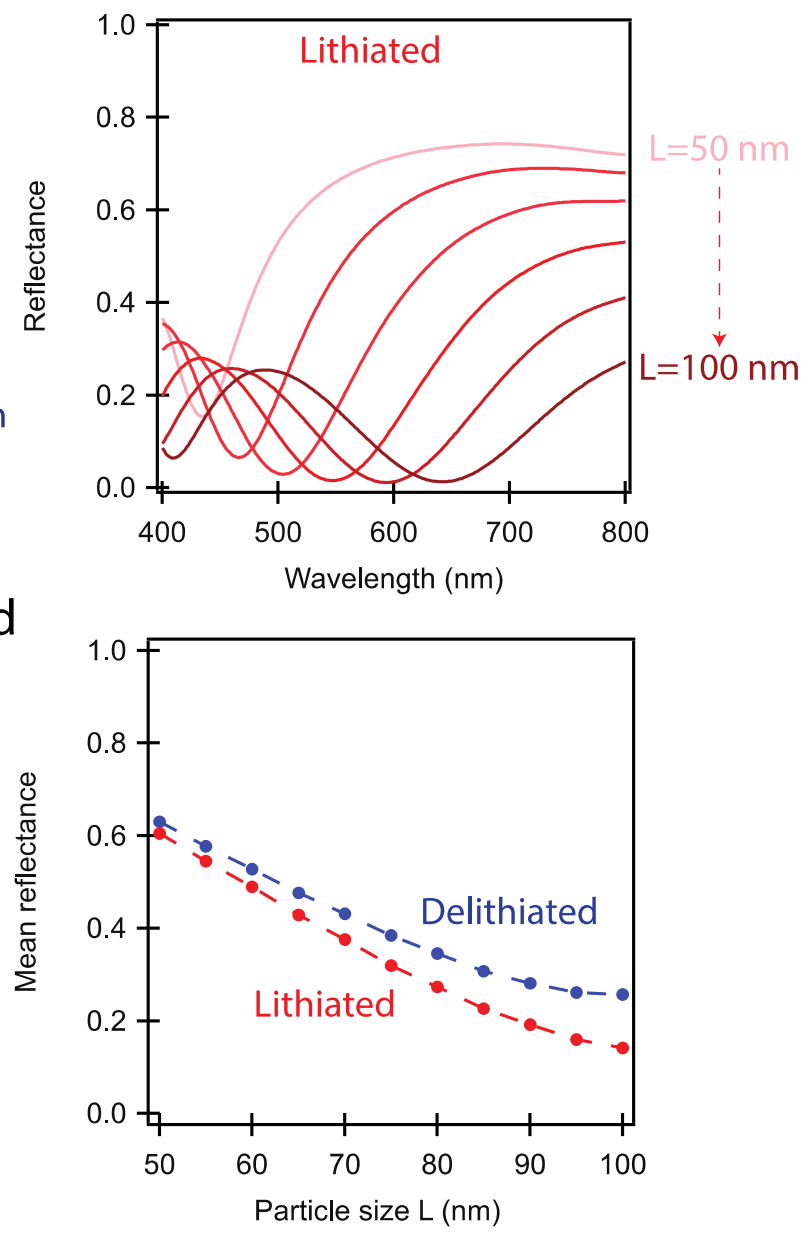

Figure S7: Simulated reflectance spectrum of the gap plasmon structures illuminated with longitudinal polarization show minimal off-resonance absorption. (a) Reflectance for the delithiated tungsten oxide structures for various particle lengths. (b) Simulated reflectance for the lithiated tungsten oxide structures. (c) A comparison of the reflectance of the lithiated and delithiated structures with $50-\mathrm{nm}$ and $100-\mathrm{nm}$ particle sizes. While the absorption in the tungsten oxide is large on and near resonance, there is little off-resonant absorption. For example, the offresonance reflectance decreases by less than 3\% for 700-nm light in 50-nm particles. (d) Mean reflectance averaged across all wavelengths between 400 and $800 \mathrm{~nm}$. At larger particle sizes, the gap plasmon resonance wavelength increases to the middle of the visible spectrum. Thus, more resonant absorption occurs in the lithiated $\mathrm{WO}_{3}$, thereby decreasing the average reflectance. The absorption at larger particle sizes is somewhat higher in simulation than in experiment. This may reflect differences in the experimental and simulated particle sizes, or differences in the optical constants of the $\mathrm{WO}_{3}$ measured through ellipsometry (Fig. 2a), which were fabricated on silicon and delithiated in liquid electrolytes, compared to the ones in the solid-state device (Fig. 1a), which were fabricated in a gap plasmon structure and reside in solid electrolytes. 


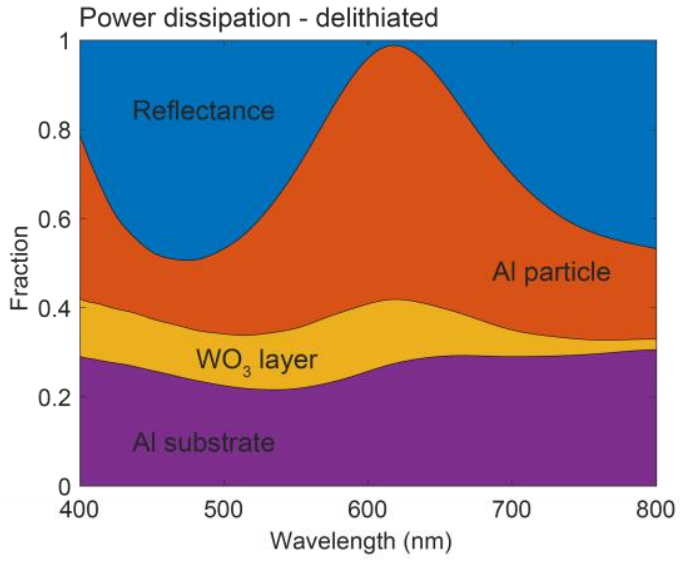

Intensity enhancement - delithiated

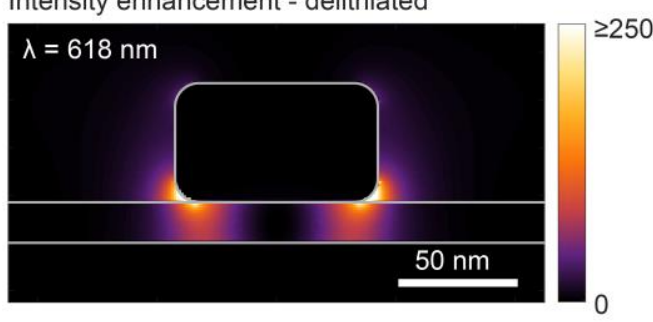

Absorption rate - delithiated

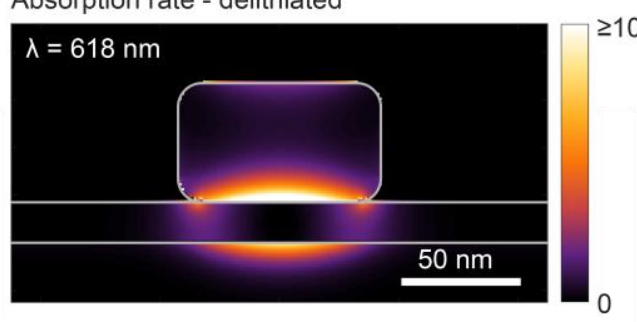

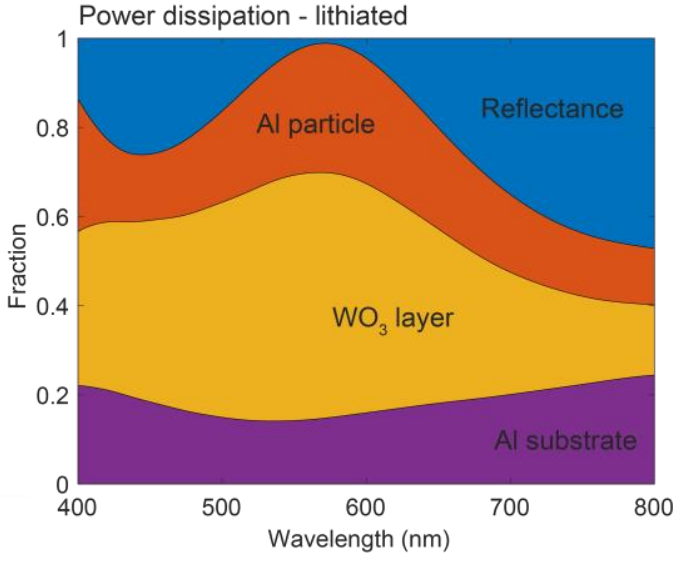

Intensity enhancement - lithiated

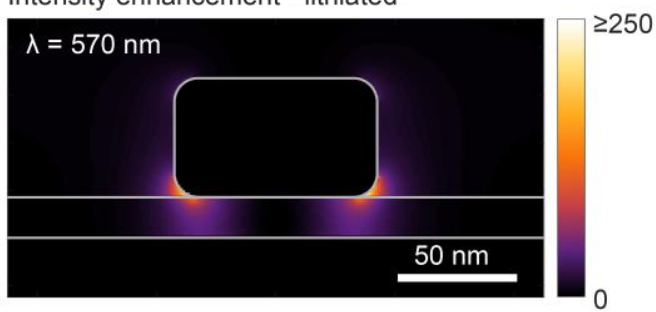

Absorption rate - lithiated

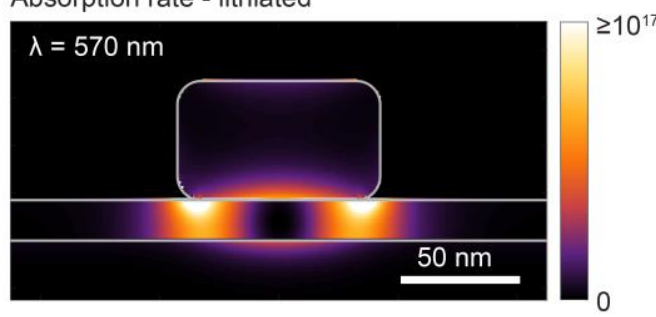

Figure S8: Simulated power dissipation in each layer and absorption profiles under illumination with longitudinal polarization for a particle with $L=85 \mathrm{~nm}$ and $W=60 \mathrm{~nm}$. (Top) The fraction of the optical power absorbed in each layer (colored area) and reflected power (blue) as a function of wavelength for the delithiated (left) and lithiated (right) $\mathrm{WO}_{3}$. The spectrally averaged absorption (425-750 $\mathrm{nm}$ to match Fig. 2) shifts from $60 \%$ in the metal and $11 \%$ in the $\mathrm{WO}_{3}$ layer for the delithiated case, to $39 \%$ in the metal and $42 \%$ in the $\mathrm{WO}_{3}$ layer in the lithiated case. (Bottom) Comparing the intensity enhancement profiles at resonance shows that besides an overall reduction in the intensity, the spatial profile remains largely unaltered upon lithiation. The spatial absorption rate profile on the other hand - calculated as $\frac{1}{2} \omega \varepsilon^{\prime \prime}|E|^{2}-$ shows a strong shift from absorption in the metal to absorption in the $\mathrm{WO}_{3}$ layer. 

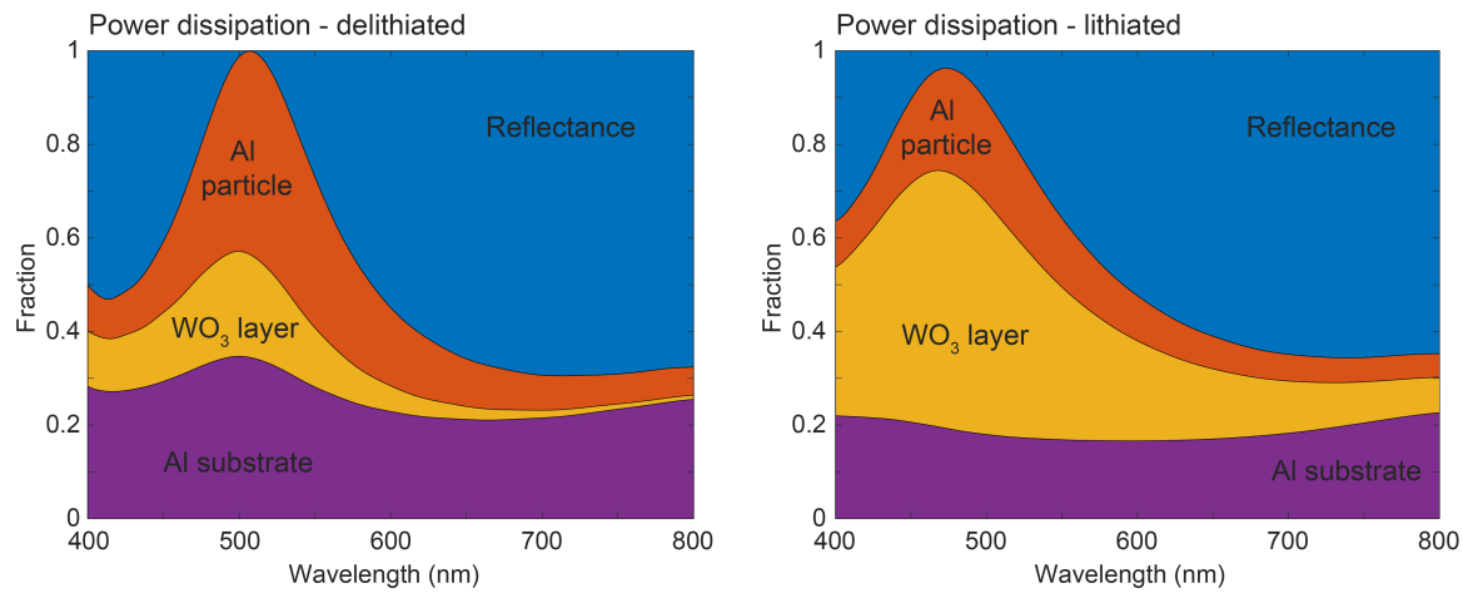

Figure S9: Simulated power dissipation in each layer under illumination with perpendicular polarization for a particle with $L=85 \mathrm{~nm}$ and $W=60 \mathrm{~nm}$. The fraction of the optical power absorbed in each layer (colored area) and reflected power (blue) as a function of wavelength for the delithiated (left) and lithiated (right) $\mathrm{WO}_{3}$. The spectrally averaged absorption (425-750 $\mathrm{nm}$ to match Fig. 2) shifts from $45 \%$ in the metal and $9 \%$ in the $\mathrm{WO}_{3}$ layer for the delithiated case, to $30 \%$ in the metal and $28 \%$ in the $\mathrm{WO}_{3}$ layer in the lithiated case.

\section{References}

1. Lumerical Solutions Inc. FDTD Solutions. Available at: http://www.lumerical.com.

2. Palik, E. D. Handbook of Optical Constants of Solids. (Academic, 1985). 